\title{
Unambiguous determination of gravitational waveforms from binary black hole mergers
}

\author{
C. Reisswig, ${ }^{1}$ N. T. Bishop, ${ }^{2,3}$ D. Pollney, ${ }^{1}$ and B. Szilágyi ${ }^{4}$ \\ ${ }^{1}$ Max-Planck-Institut für Gravitationsphysik, Albert-Einstein-Institut, 14476 Golm, Germany \\ ${ }^{2}$ Department of Mathematics, Rhodes University, Grahamstown 6140, South Africa \\ ${ }^{3}$ Department of Mathematical Sciences, University of South Africa, Unisa 0003, South Africa \\ ${ }^{4}$ Theoretical Astrophysics, California Institute of Technology, Pasadena, CA 91125, USA
}

\begin{abstract}
Gravitational radiation is properly defined only at future null infinity $\left(\mathcal{J}^{+}\right)$, but in practice it is estimated from data calculated at a finite radius. We have used characteristic extraction to calculate gravitational radiation at $\mathcal{J}^{+}$for the inspiral and merger of two equal mass non-spinning black holes. Thus we have determined the first unambiguous merger waveforms for this problem. The implementation is general purpose, and can be applied to calculate the gravitational radiation, at $\mathcal{J}^{+}$, given data at a finite radius calculated in another computation.
\end{abstract}

PACS numbers: 04.25.dg, 04.30.Db, 04.20.Ha, 04.30.Nk

Introductory Remarks. The computation of gravitational radiation from black hole merger events has attracted considerable attention, since the pioneering work by Smarr and collaborators [1-3]. With the advent of ground-based laser interferometric gravitational wave detectors, as well as the prospect of the Laser Interferometer Space Antenna (LISA), interest in the problem has considerably increased. The measurement of gravitational waves will soon provide an important probe of strong-field nonlinear gravity, the domain of many fundamental questions in astrophysics. The sensitivity of LISA, and of the upcoming advanced ground-based detectors AdLIGO and AdVirgo, is so high that even an error in the waveform calculation of $0.1 \%$ (in a sense made precise later) could lead to an incorrect interpretation of the astrophysical properties of a source, or of a test of general relativity. Nowadays, there are several codes that can produce a stable and convergent simulation of a black hole spacetime. However, a particular difficulty with measuring gravitational radiation arises from the fact that, in general relativity, it cannot be defined locally but is defined only at future null infinity $\left(\mathcal{J}^{+}\right)$, which, physically, is the limit that is approached by radiation moving at the speed of light away from an isolated source. Since numerical evolutions are normally carried out on finite domains, there is a systematic error caused by estimating the gravitational radiation from fields on a worldtube at finite radius and the uncertainty in how it relates to measurement at $\mathcal{J}^{+}$[4]. Even if this error is small, the expected sensitivity of AdLIGO, AdVirgo and LISA implies that it is important to obtain an accurate result.

A rigorous formalism for the global measurement of gravitational energy at null infinity has been in place since the pioneering work of Bondi, Penrose and collaborators in the 1960s [5, 6]; and subsequently, techniques for calculating gravitational radiation at $\mathcal{J}^{+}$have been developed. The idea which we pursue here is to combine a Cauchy or " $3+1$ " numerical relativity code with a characteristic code [7]. Given astrophysical initial data, such a method has only discretization error [8], and a complete mathematical specification has been developed [9]. There have been efforts to implement this method, often called Cauchy characteristic extraction (CCE), or characteristic extraction [10, 11]. Previous work has considered test problems rather than that of the inspiral and merger of two black holes or neutron stars. Also, earlier efforts have combined the Cauchy and characteristic algorithms within the same code.

Here, we describe the implementation of a CCE code as well as results obtained from the code for the inspiral and merger waveform of two equal mass, non-spinning, black holes. The waveforms are calculated at $\mathcal{J}^{+}$, and are thus the first unambiguous waveforms which have been obtained for this problem, in the sense of being free of gauge or finiteradius effects. Further, the code is general purpose, in that it is independent of the details of the Cauchy code, requiring only that it prescribes the required geometrical data on a worldtube. Thus its application to other astrophysical problems will be straightforward.

For the specific problem of a binary black hole $(\mathrm{BBH})$ merger, we show that the waveform obtained at $\mathcal{J}^{+}$contains only numerical error and is gauge-invariant. We demonstrate second-order convergence to zero in the amplitude and phase differences between two CCE runs using boundary data at different radii. We compare the waveform obtained at $\mathcal{J}^{+}$with a finite-radius extrapolated waveform, and find that the corrections introduced by CCE are visible in the groundbased detectors AdLIGO and AdVirgo, as well as the space-based LISA detector.

Cauchy Evolution. The scenario we envision is an isolated system (perturbed single body, or gravitationally bound binary), in a region on which the Einstein (and possibly hydrodynamic) equations must be solved. A standard procedure for doing this is to formulate the equations as an initial-boundaryvalue, or Cauchy, problem, in which data for the 3-metric and its embedding is prescribed at a given time on a closed region of the spacetime, $\Sigma_{t}$. These are evolved according to the Einstein equations on the interior of the domain, and artificial conditions on the timelike boundary, $\partial \Sigma_{t}$. The first stable evolutions of a binary black hole system were carried out by Pretorius [12]. Two approaches to the evolution of the interior equations are in use: (a) the harmonic formulation of the Einstein equations with excised black hole interiors [12, 13]; (b) the BSSNOK (see [14] and references therein) evolution system with the black holes specified as moving "punctures" [15, 16].

For the Cauchy evolutions used here, we have followed the latter approach using the formulation outlined in [17]. The spacetime is discretised using finite differences on Cartesian grids and Berger-Oliger mesh refinement in the neighbour- 
hood of the black holes [18]. The wave zone is discretised by six overlapping coordinate patches with spherical topology. Interior boundary data between adjacent patches are communicated by interpolation [19, 20].

The outer boundary condition on the exterior of the domain is given by a linear outgoing wave condition on each of the evolved tensor components. Importantly, through the use of spherical grids in the wave zone, a sufficient resolution can be maintained even to a distant outer boundary, reducing the effect of grid reflections common in mesh-refinement codes. The size of the evolution domain is chosen according to the amount of time required, $T$, and the location of the outermost measurement sphere, $r_{i}$. Since physical as well as constraint violating modes propagate with the speed of light, an outer boundary located at $r_{\partial \Sigma_{t}}>T+r_{i}$ ensures that measurements are causally disconnected from the influence of the outer boundary.

Characteristic extraction. Our implementation of CCE is based on the mathematical prescription given in [9], and here we provide only an outline. The process is illustrated schematically in Fig. 11 Within a Cauchy simulation that uses Minkowski-like coordinates $(t, x, y, z)$, we define a worldtube $\Gamma$ by $x^{2}+y^{2}+z^{2}=r_{\Gamma}^{2}$, and compute the lapse $\alpha$, the shift $\beta^{i}$ and the 3-metric $\gamma_{i j}$ on $\Gamma$, as well as their first time and radial derivatives. This data is then decomposed into spherical harmonics. The Cauchy code writes this spherical harmonic coefficient data to file, and later the CCE code postprocesses the data to reconstruct the 4-metric on the inner world tube. In this way, the CCE code is general purpose, as it runs independently of whatever Cauchy code was used to generate the worldtube data.

The CCE code defines angular coordinates $\phi^{A}$ as well as a time coordinate $u(=t)$ on $\Gamma$, and constructs outgoing null geodesics with affine parameter $\lambda$. It then transforms the Cauchy 4-metric to $\left(u, \lambda, \phi^{A}\right)$ coordinates, and calculates a surface area radial coordinate $r_{S}$, making the coordinate transformation to $\left(u, r_{S}, \phi^{A}\right)$ coordinates, in order to obtain the Bondi-Sachs metric data in a neighbourhood of $\Gamma$. This provides the inner boundary data for the characteristic evolution, using a Cactus [21] implementation of the PITT null evolution code with square stereographic coordinate patches [22]. The characteristic code uses coordinates based on outgoing null cones, and so the equations remain regular when the radial coordinate is compactified (by $r_{S} \rightarrow z=r_{S} /\left(r_{S}+r_{S \Gamma}\right)$ ), and in this way $\mathcal{J}^{+}$is included on the computational grid. The code computes the gravitational radiation at $\mathcal{J}^{+}$as the Weyl component $\psi_{4}$. The coordinate-independent quantity $\psi_{4}$ is commonly used in numerical relativity; in appropriate coordinates, it is the second time derivative of the strain measured by a detector.

Binary black hole evolution. We have carried out fully relativistic evolutions of an equal-mass non-spinning binary black hole inspiral and merger. The initial data parameters for the closely bound black hole are determined by performing a postNewtonian evolution from large separation in order to determine the momenta for low-eccentricity (quasi-circular) trajectories [23]. The subsequent full nonlinear numerical relativity evolution proceeds for approximately 8 orbits $(1350 M)$, fol-

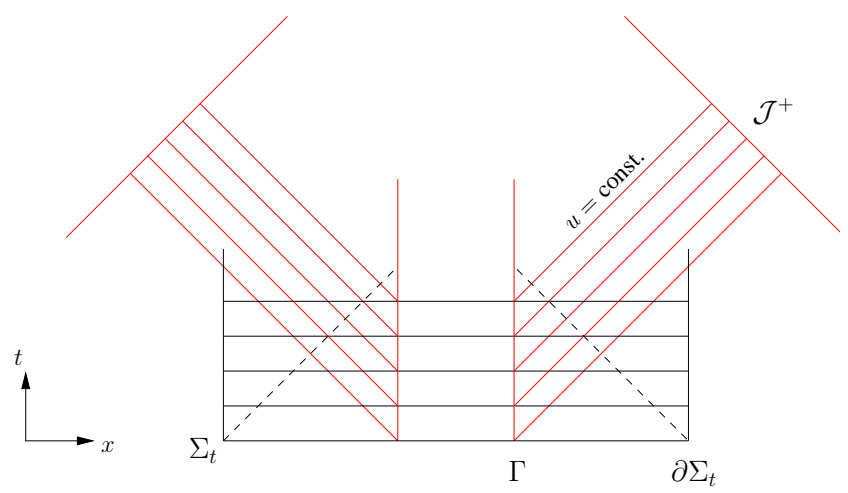

FIG. 1: Schematic of the CCE algorithm with two spatial dimensions suppressed. Spacelike slices, $\Sigma_{t}$ are evolved according to the Cauchy evolution scheme (horizontal lines). Geometrical data is recorded on a world-tube, $\Gamma$, which is used as interior boundary data for a characteristic evolution along $u=$ constant null surfaces, transporting the data to $\mathcal{J}^{+}$. The outer boundary of the Cauchy domain, $\partial \Sigma_{t}$ is chosen so that it is causally disconnected from $\Gamma$ over the course of the evolution.

lowed by the merger and ringdown lasting another $100 \mathrm{M}$.

The evolutions have been carried out at two different grid resolutions in order to verify the convergence of the numerical scheme. The grid settings for the Cauchy code are: The central Cartesian grid consists of 6 levels of 2:1 mesh-refinement, with coarse grid spacings of $h=0.96 M$ and $h=0.64 M$, respectively. A grid of spherical topology covers the far field, $r \in[35 M, 3600 M]$; so that, during the time period of interest, the outer boundary is causally disconnected from any extraction sphere (see Fig. 11). The radial spacing is commensurate with the coarse Cartesian grid at the interface, and (for reasons of efficiency) is gradually scaled to $h=3.84 M$ and $h=2.56 \mathrm{M}$ at the outer boundary for the two runs. We use a corresponding $N_{\text {ang }}=21$ and $N_{\text {ang }}=31$ points in each of the angular directions per patch.

Characteristic boundary data were interpolated onto worldtubes located at $r=100 M$ and $r=200 M$, and stored in the form of spherical harmonic coefficients, up to $\ell=8$, which was found to be the highest resolved mode. The resolutions of the characteristic evolutions are set up according to the respective resolutions of the Cauchy run. We use $N_{r}=321$ and $N_{r}=481$ radial points, with $N_{\text {ang }}=51$ and $N_{\text {ang }}=76$ angular points per angular patch. The dominant $\ell=2, m=2$ mode of the gravitational waveform resulting from the numerical evolution is plotted in Fig. 2 to be described in more detail below.

Invariance with respect to the world tube location is demonstrated in Fig 3 . We have considered the differences between waveforms at $\mathcal{J}^{+}$resulting from two independent characteristic evolutions using boundary data at $r_{\Gamma}=100 \mathrm{M}$ and $r_{\Gamma}=$ $200 M$, respectively, and for two resolutions, $h=0.96 M$ and $h=0.64 M$. The difference between the results should be entirely due to the discretisation error, and indeed this is what we find. The differences converge to zero with approximately second-order accuracy, as expected for the null evo- 

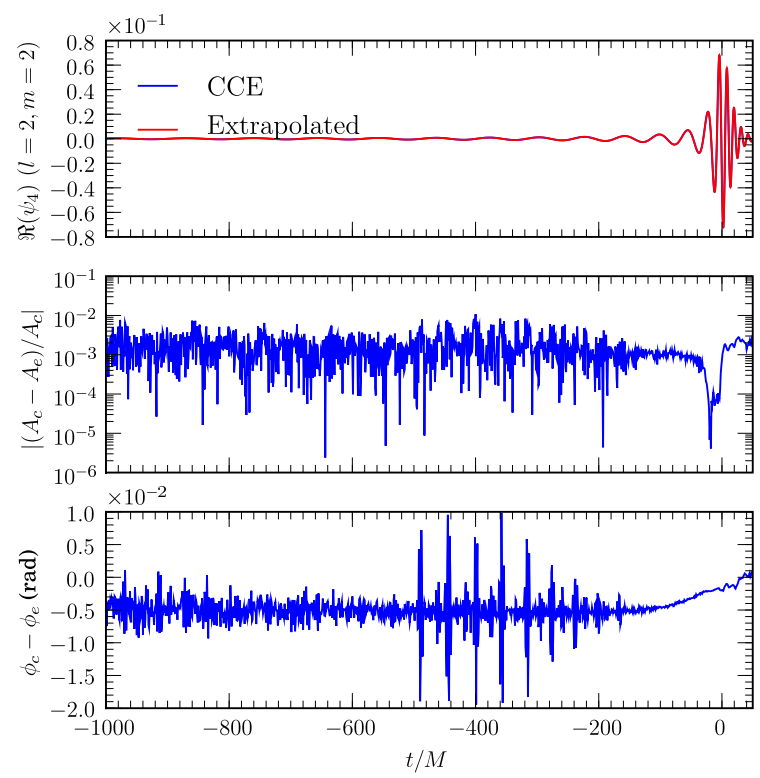

FIG. 2: Inspiral, merger and ringdown phase of $\psi_{4}(\ell=2, m=2)$ as obtained from finite radius extrapolation (red) and at $\mathcal{J}^{+}$(blue). The waveforms are aligned at their peaks. There is a maximum difference of $1.08 \%$ in the amplitude and a dephasing of 0.019 radians between the two waves. These differences can introduce systematic errors to parameter estimation of events detected at high SNR.

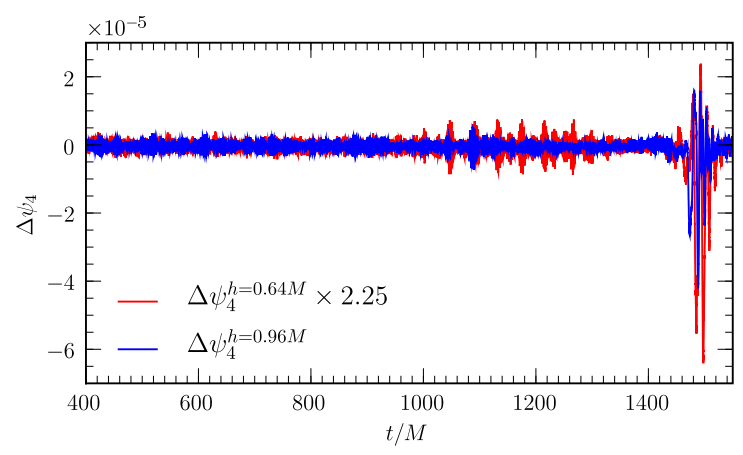

FIG. 3: Differences $\Delta \psi_{4}$ in the amplitude of $\psi_{4}(\ell=2, m=2)$ between two characteristic runs using boundary data from $R_{\Gamma}=$ $100 M$ and $R_{\Gamma}=200 M$. The red curve shows the difference at resolution $h=0.96 \mathrm{M}$ while the blue curve shows the difference for $h=0.64 M$, scaled so as to line up for second-order convergence. The expected second order convergence of our code is thus demonstrated.

lution code. The figure displays the differences $\Delta \psi_{4}$ in the amplitude of the wave mode $\psi_{4}(\ell=2, m=2)$ for resolution $h=0.96 M$ and $h=0.64 M$ scaled for second-order convergence. The same order of convergence is also obtained for higher order modes such as $\psi_{4}(\ell=4, m=4)$ (not displayed). The differences between the waveforms at $\mathcal{J}^{+}$for resolution $h=0.64 M$ are of order of $0.03 \%$ in amplitude with a dephasing of 0.002 radians.

The CCE waves can be used to evaluate the quality of standard finite radius measurements, extrapolated to $r \rightarrow \infty$; previously, this was the most accurate option available. We do so by finding the Weyl component $\psi_{4}$ relative to a radially oriented null tetrad [17] (we prefer $\psi_{4}$ to gauge-invariant perturbative methods [24-26]). We have evaluated $\psi_{4}$ at six radii $(r=280,300,400,500,600,1000 M)$ and extrapolated. Details are given in [19], and the error is estimated as $0.03 \%$ in amplitude and 0.003 radians in phase.

In Fig. 2, we compare the extrapolated waveform with that calculated at $\mathcal{J}^{+}$via CCE. The differences between the two waveforms have maximum and mean values of $1.08 \%$ and $0.166 \%$ in amplitude, and -0.019 and -0.004 radians in phase, respectively. That is, for the resolutions used, the numerical error in the characteristic evolution (see Fig. 3) is smaller by one order of magnitude than the error between the extrapolated and characteristic waveforms in both amplitude and phase. Further, we note that the estimated error in the extrapolation is itself much smaller than the actual error between characteristic waveform and extrapolated waveform. This indicates that the systematic error in extrapolation has, previously, been underestimated. The correction is towards slightly larger amplitudes and frequencies. CCE post-processes data produced by a Cauchy code, and as such there is an additional cost. However, it is relatively small: the Cauchy run reported here took $\sim 336$ hours, and then CCE required $\sim 10$ hours.

Will the small correction to waveforms introduced by CCE be relevant to interpreting observational data? The answer will depend on the signal-to-noise ratio (SNR) of the event. At low SNR, whether CCE or extrapolated waveforms are used as a template will not affect physical interpretation. This is particularly relevant, as numerical waveforms are being constructed with the intention of evaluating and parametrising detector templates and search algorithms [27], and to constrain analytic models [28-31]. Our results indicate that extrapolations from a finite radius can be used to construct detector templates well within the accuracy standards required by matched filtering algorithms.

However, at large SNR, the differences are significant to the determination of the physical parameters of a model measured in detector data. To demonstrate this, we follow methods described in [32, 33] to determine the minimum SNR needed for a detected signal from a merger event to lead to different parameter estimates depending on which waveform is used as a template. Table【displays the results for selected masses, indicating the maximum distance at which the difference between the waveforms will be relevant for the given merger event.

The difference between the waveforms is unlikely to be relevant for LIGO, (e)LIGO and Virgo. Reasonable stellar mass black hole merger rates are expected only for a volume encompassing sources up to a distance of at least $100 \mathrm{Mpc}$. Thus, there may well be events detected by AdLIGO and AdVirgo for which the difference is important. Finally, the differences will certainly be relevant for LISA as they will be applicable to any supermassive black hole merger event throughout the visible universe $\left(\mathrm{cH}^{-1}\right.$ is the Hubble radius).

Acknowledgments. The authors thank Stanislav Babak, Luciano Rezzolla and Jeffrey Winicour for their helpful input. CR, DP and BS thank the University of South Africa, and NTB thanks Max-Planck-Institut für Gravitationsphysik, for hospitality. This work was supported by 


\begin{tabular}{l|ll}
\hline \hline Detector & Masses & Maximum distance \\
\hline LIGO & $50 M_{\odot}+50 M_{\odot}$ & $5 \mathrm{Mpc}$ \\
(e)LIGO & $50 M_{\odot}+50 M_{\odot}$ & $8 \mathrm{Mpc}$ \\
Virgo & $50 M_{\odot}+50 M_{\odot}$ & $14 \mathrm{Mpc}$ \\
AdLIGO & $50 M_{\odot}+50 M_{\odot}$ & $197 \mathrm{Mpc}$ \\
AdVirgo & $50 M_{\odot}+50 M_{\odot}$ & $177 \mathrm{Mpc}$ \\
LISA & $10^{7} M_{\odot}+10^{7} M_{\odot}>c H^{-1}$ \\
\hline \hline
\end{tabular}

TABLE I: Maximum distance at which the difference between the extrapolated waveform and that at $\mathcal{J}^{+}$would be significant for a black hole merger event.
National Research Foundation, South Africa; Budesministerium für Bildung und Forschung, Germany; and DFG grant SFB/Transregio 7 "Gravitational Wave Astronomy". DP was supported by a grant from the VESF. BS was supported by grants from the Sherman Fairchild Foundation, by NSF grants DMS-0553302, PHY-0601459, PHY-0652995, and by NASA grant NNX09AF97G. Computations were performed at the AEI, at LRZ-München, on Teragrid clusters (allocation TGMCA02N014), and LONI resources at LSU.
[1] L. Smarr, Ph.D. thesis, University of Texas, Austin, Austin, Texas (1975).

[2] L. Smarr, A. Čadež, B. DeWitt, and K. R. Eppley, Phys. Rev. D 14, 2443 (1976).

[3] L. Smarr and J. W. York, Phys. Rev. D 17, 2529 (1978).

[4] B. Kocsis and A. Loeb, Physical Review D 76, 084022 (2007), 0704.1149.

[5] H. Bondi, M. G. J. van der Burg, and A. W. K. Metzner, Proc. R. Soc. London A269, 21 (1962).

[6] R. Penrose, Phys. Rev. Lett. 10, 66 (1963).

[7] N. T. Bishop, Class. Quantum Grav. 10, 333 (1993).

[8] N. T. Bishop, R. Gómez, L. Lehner, and J. Winicour, Phys. Rev. D 54, 6153 (1996).

[9] N. Bishop, R. Isaacson, R. Gómez, L. Lehner, B. Szilágyi, and J. Winicour, in Black Holes, Gravitational Radiation and the Universe, edited by B. Iyer and B. Bhawal (Kluwer, Dordrecht, The Neterlands, 1999), p. 393.

[10] M. Babiuc, B. Szilágyi, I. Hawke, and Y. Zlochower, Class. Quantum Grav. 22, 5089 (2005), gr-qc/0501008.

[11] M. C. Babiuc, N. T. Bishop, B. Szilágyi, and J. Winicour, Phys. Rev. D79, 084011 (2009), gr-qc/0808.0861.

[12] F. Pretorius, Phys. Rev. Lett. 95, 121101 (2005), grqc/0507014.

[13] M. A. Scheel et al., Phys. Rev. D79, 024003 (2009), 0810.1767.

[14] M. Alcubierre, Introduction to $3+1$ Numerical Relativity (Oxford University Press, Oxford, UK, 2008).

[15] J. G. Baker, J. Centrella, D.-I. Choi, M. Koppitz, and J. van Meter, Phys. Rev. Lett. 96, 111102 (2006), gr-qc/0511103.

[16] M. Campanelli, C. O. Lousto, P. Marronetti, and Y. Zlochower, Phys. Rev. Lett. 96, 111101 (2006), gr-qc/0511048.

[17] D. Pollney, C. Reisswig, L. Rezzolla, B. Szilágyi, M. Ansorg, B. Deris, P. Diener, E. N. Dorband, M. Koppitz, A. Nagar, et al.,
Phys. Rev. D76, 124002 (2007), arXiv:0707.2559.

[18] E. Schnetter, S. H. Hawley, and I. Hawke, Class. Quantum Grav. 21, 1465 (2004), gr-qc/0310042.

[19] D. Pollney, C. Reisswig, N. Dorband, E. Schnetter, and P. Diener (2009), arXiv:0910.3656 [gr-qc].

[20] D. Pollney, C. Reisswig, E. Schnetter, N. Dorband, and P. Diener (2009), arXiv:0910.3803 [gr-qc].

[21] G. Allen, T. Goodale, and E. Seidel, in 7th Symposium on the Frontiers of Massively Parallel Computation-Frontiers 99 (IEEE, New York, 1999), Annapolis, Maryland.

[22] N. T. Bishop, R. Gómez, L. Lehner, M. Maharaj, and J. Winicour, Phys. Rev. D 56, 6298 (1997), gr-qc/9708065.

[23] S. Husa, M. Hannam, J. A. Gonzalez, U. Sperhake, and B. Bruegmann, Phys. Rev. D77, 044037 (2008), 0706.0904.

[24] V. Moncrief, Annals of Physics 88, 323 (1974).

[25] F. J. Zerilli, Phys. Rev. Lett. 24, 737 (1970).

[26] A. Nagar and L. Rezzolla, Class. Quant. Grav. 22, R167 (2005), gr-qc/0502064.

[27] B. Aylott et al., Class. Quant. Grav. 26, 165008 (2009), 0901.4399.

[28] A. Buonanno et al., Phys. Rev. D79, 124028 (2009), 0902.0790.

[29] T. Damour and A. Nagar (2009), 0902.0136.

[30] P. Ajith, S. Babak, Y. Chen, M. Hewitson, B. Krishnan, J. T. Whelan, B. Brügman, J. Gonzalez, M. Hannam, S. Husa, et al., Phys. Re. D 77, 104017 (2008), arXiv:0710.2335.

[31] P. Ajith et al. (2009), 0909.2867.

[32] L. Lindblom, B. J. Owen, and D. A. Brown, Phys. Rev. D78, 124020 (2008), 0809.3844.

[33] M. Hannam et al., Physical Review D 79, 084025 (2009), 0901.2437. 